Ferrata Storti Foundation

\section{Specialized pro-resolving lipid mediators are differentially altered in peripheral blood of patients with multiple sclerosis and attenuate monocyte and blood-brain barrier dysfunction}

\author{
Gijs Kooij,,$^{1,2^{*}}$ Claudio Derada Troletti, ${ }^{1 *}$ Alessandro Leuti, ${ }^{3,4}$ Paul C. Norris, ${ }^{2}$ \\ Ian Riley, ${ }^{2}$ Maria Albanese, ${ }^{5}$ Serena Ruggieri, ${ }^{6}$ Stephania Libreros, ${ }^{2}$ \\ Susanne M.A. van der Pol, ${ }^{1}$ Bert van het Hof, ${ }^{1}$ Yoëlle Schell, ${ }^{1}$ \\ Gisella Guerrera, ${ }^{4}$ Fabio Buttari, ${ }^{7}$ Nicola Biagio Mercuri, ${ }^{4,5}$ Diego Centonze, ${ }^{5,7}$ \\ Claudio Gasperini, ${ }^{6}$ Luca Battistini, ${ }^{4}$ Helga E. de Vries, ${ }^{1}$ Charles N. Serhan, ${ }^{2 \#}$ \\ Valerio Chiurchiừ ${ }^{3,4}$
}

Volume 105(8):2056-2070

\begin{abstract}
${ }^{1}$ Amsterdam UMC, Vrije Universiteit Amsterdam, Department of Molecular Cell Biology and Immunology, MS Center Amsterdam, Amsterdam Neuroscience, Amsterdam, the Netherlands; ${ }^{2}$ Center for Experimental Therapeutics and Reperfusion Injury, Department of Anesthesiology, Perioperative and Pain Medicine, Brigham and Women's Hospital and Harvard Medical School, Boston, MA, USA; ${ }^{3}$ Department of Medicine, Campus Bio-Medico University of Rome, Rome, Italy; ${ }^{4}$ European Center for Brain Research, IRCCS Santa Lucia Foundation, Rome, Italy; ${ }^{5}$ Neurology Unit, Department of Neurosciences, University of Rome Tor Vergata, Rome, Italy; ${ }^{6}$ Neurology Unit, San Camillo Forlanini Hospital, Rome, Italy and ${ }^{7}$ Unit of Neurology and Unit of Neurorehabilitation, IRCCS Istituto Neurologico Mediterraneo (INM) Neuromed, Pozzilli, IS, Italy
\end{abstract}

* GK and CDT contributed equally as first authors.

"CNS and VC contributed equally as co-senior authors.

\section{Correspondence:}

VALERIO CHIURCHIÙ

v.chiurchiu@hsantalucia.it

CHARLES N. SERHAN

cserhan@bwh.harvard.edu

Received: February 13, 2019.

Accepted: November 21, 2019.

Pre-published: November 28, 2019.

doi:10.3324/haematol.2019.219519

Check the online version for the most updated information on this article, online supplements, and information on authorship \& disclosures: www. haematologica.org/content/105/8/2056

\section{(C)2020 Ferrata Storti Foundation}

Material published in Haematologica is covered by copyright. All rights are reserved to the Ferrata Storti Foundation. Use of published material is allowed under the following terms and conditions:

https://creativecommons.org/licenses/by-nc/4.0/legalcode. Copies of published material are allowed for personal or internal use. Sharing published material for non-commercial purposes is subject to the following conditions:

https://creativecommons.org/licenses/by-nc/4.0/legalcode, sect. 3. Reproducing and sharing published material for commercial purposes is not allowed without permission in writing from the publisher.

\section{ABSTRACT}

hronic inflammation is a key pathological hallmark of multiple sclerosis (MS) and suggests that resolution of inflammation, orchestrated by specialized pro-resolving lipid mediators (LM), is impaired. Here, through targeted-metabololipidomics in peripheral blood of patients with MS, we revealed that each disease form was associated with distinct LM profiles that significantly correlated with disease severity. In particular, relapsing and progressive MS patients were associated with high eicosanoids levels, whereas the majority of pro-resolving LM were significantly reduced or below limits of detection and correlated with disease progression. Furthermore, we found impaired expression of several pro-resolving LM biosynthetic enzymes and receptors in blood-derived leukocytes of MS patients. Mechanistically, differentially expressed mediators like LXA $\mathrm{LXB}_{4}, \mathrm{RvD} 1$ and $\mathrm{PD} 1$ reduced MS-derived monocyte activation and cytokine production, and inhibited inflammation-induced blood-brain barrier dysfunction and monocyte transendothelial migration. Altogether, these findings reveal peripheral defects in the resolution pathway in MS, safe therapeutics.

\section{Introduction}

Multiple sclerosis (MS) is the most common chronic inflammatory demyelinating disease of the central nervous system (CNS) associated to uncontrolled/excessive neuro-inflammation and autoimmunity. ${ }^{1,2}$ The underlying immunopathogenesis of the disease has been extensively studied and is currently thought to involve an initial alteration of peripheral and brain immune responses, as well as a disruption of the blood-brain barrier (BBB). Subsequently, this leads to a substantial infiltration of autoreactive lymphocytes and innate immune cells causing demyelination, axonal loss, and ultimately neurodegeneration. ${ }^{3.6}$ Nevertheless, there is still an unmet need for new diagnostic and therapeutic options, especially for the progressive forms of MS for which almost no drugs are available (with the exception of the offlabel rituximab and the recently approved ocrelizumab for the management of prisuggesting pro-resolving LM as novel diagnostic biomarkers and potentially 
mary progressive MS). Recent studies suggest that chronic inflammation and autoimmunity could be a consequence of failure to resolve inflammation, and this resolution of inflammation is mediated by newly discovered metabolites termed specialized pro-resolving lipid mediators (SPM), temporally and spatially synthesized from $\omega-3$ polyunsaturated fatty acids [eicosapentaenoic acid (EPA) and docosahexaenoic acid (DHA)]. ${ }^{7}$ During the process of resolution of inflammation, the very same cells recruited to the inflammatory milieu and that produce inflammatory mediators (mainly innate immune cells) undergo a temporal lipid mediator (LM) class switch, whereby they stop producing classical eicosanoids (prostaglandins, leukotrienes, thromboxanes) from $\omega-6$ arachidonic acid and start to biosynthesize SPM mainly from $\omega-3$ EPA and DHA,, 78 through the stereoselective and concerted action of the same enzymes engaged in classical eicosanoids production, namely cyclo-oxygenase (COX) COX-2, lipoxygenases (LOX) LOX-5, LOX-12 and LOX-15, as well as cytochrome P450 and several pathway specific epoxide hydrolases. These LM are potent and extinguish the eicosanoid-induced inflammation by activating local resolution programs ${ }^{7-9}$ also by directly modulating oxidative stress ${ }^{10}$ and $T$-cell responses, ${ }^{11}$ via five separate $\mathrm{G}$ proteincoupled receptors (e.g. ALX/FPR2, GPR32/DRV1, ChemR23/ERV, BLT1 and GPR18/DRV2), ${ }^{9}$ without evoking unwanted side effects as opposed to the immunosuppressive agents that are currently most used as diseasemodifying treatments. Despite the increase in data suggesting that SPM metabolism and functions are differentially altered in several chronic peripheral and brain inflammatory diseases, ${ }^{7,10,12,13}$ research on these LM in MS and how they contribute to disease is of high interest. Given this, we aimed to determine whether MS patients at different phases of disease, compared to healthy subjects, displayed different levels and abilities to endogenously synthesize and respond to $\omega-6$ - and $\omega$-3-derived pro-inflammatory (eicosanoids) and pro-resolving LM (lipoxins, resolvins, protectins and maresins) by means of targeted lipid metabololipidomics in human plasma and by evaluating the expression of their enzymes and key target receptors in peripheral blood mononuclear cells (PBMC). Finally, we investigated whether specific SPM could modulate the inflammatory response of MS patientderived monocytes and whether they could attenuate inflammation-induced BBB dysfunction as well as monocyte-transendothelial migration, which all represent key pathological hallmarks of MS pathogenesis. ${ }^{14}$

\section{Methods}

\section{Multiple sclerosis patients}

Peripheral blood was collected from two different cohorts. The first cohort was admitted to the neurological clinic of the University Hospital Tor Vergata, Rome, (14 females and 6 males, mean age $34.51 \pm 3.35$ years) and the second cohort to the San Camillo Hospital of Rome (13 females and 5 males, mean age $38.24 \pm 2.76$ years). Both cohorts were diagnosed as suffering from relapsing-remitting MS (RR-MS, $\mathrm{n}=26$ ) or primary progressive $(\mathrm{P}-\mathrm{MS}, \mathrm{n}=12)$. Fifteen age-matched healthy subjects $(\mathrm{HS}, \mathrm{n}=15)$ were used as controls. See Table 1 and Online Supplementary Methods for diagnostic details. All subjects gave their written informed consent to the study which was approved by the ethics committees of Tor Vergata Hospital and of San Camillo Hospital, Rome.

\section{Liquid chromatography-tandem mass spectrometry-based metabololipidomics and analysis}

Total lipids were extracted from plasma samples with solid phase C18 cartridges. Liquid chromatography-tandem mass spectrometry (LC-MS-MS) was used to perform absolute quantifications of all LM. ${ }^{15,16}$ Lipidomics data were analyzed by principal component analysis using SIMCA 13.0.3 software (MKS Data Analytics Solution Umea, Sweden) and by volcano plots using MetaboAnalyst (http://www. metaboanalyst.ca).

\section{Human leukocyte and brain endothelial cell treatments}

Freshly isolated PBMC from HS or MS patients were left untreated or pretreated with SPM and then stimulated with Imiquimod and ssRNA40 for five hours in presence of brefeldin A. ${ }^{17-19}$ Human brain endothelial cell line hCMEC/D3 cells were grown and treated with TNF- $\alpha$ in the presence or absence of SPM $^{20}$

\section{Flow cytometry}

Peripheral blood mononuclear cells were assayed for surface immunophenotype (CD14, CD16, CD69) and intracellular cytokine production (TNF- $\alpha$, IL-1 $\beta$, IL- 6 and IL-12) by multiple fluorochrome-conjugated antibody staining. hCMEC/D3 were assayed for anti-ICAM-1 (REK-1) and SPM receptors (GPR32, ALX/FPR2 and GPR18) through primary specific antibodies followed by fluorochrome-conjugated secondary antibodies. ${ }^{11,17}$

\section{Real-time quantitative polymerase chain reaction}

Total RNA was extracted from PBMC and hCMEC/D3, and retro-transcribed to cDNA. Specific probes for SPM receptors and SPM biosynthetic enzymes were used to assess relative mRNA

Table 1. Demographic data of patients with multiple sclerosis (MS) and control subjects.

\begin{tabular}{|c|c|c|c|c|}
\hline & Healthy & Relapsing MS & Remitting MS & Progressive MS \\
\hline N. of subjects & $\mathrm{n}=14$ & $\mathrm{n}=14$ & $\mathrm{n}=12$ & $n=12$ \\
\hline Mean age & $36.12 \pm 1.77$ & $36.82 \pm 2.91$ & $37.67 \pm 2.23$ & $38.82 \pm 2.54$ \\
\hline Female/male & $9 / 6$ & $10 / 4$ & $9 / 3$ & $8 / 4$ \\
\hline Disease duration* (years) & - & 3.6 & 4.2 & 6.1 \\
\hline Mean EDSS & - & $<3(1.5-3)$ & $<3(1-1.5)$ & $>3(4-6)$ \\
\hline Corticosteroids" (yes/no) & 0 & $0 / 0$ & $0 / 0$ & $0 / 0$ \\
\hline $\mathrm{DMT}^{\sharp}$ (yes/no) & 0 & $0 / 0$ & $0 / 0$ & $0 / 0$ \\
\hline N. of Gd+ T2 brain MRI lesions (\%) & 0 & $10-20(65 \%)$ & $10-20(45 \%)$ & $>20(50 \%)$ \\
\hline
\end{tabular}

*Disease duration was defined as the time from disease onset to the time of sampling (in years).\#At time of sampling. EDSS: Expanded Disability Status Scale scores; DMT: disease modifying treatments; Gd: gadolinium; MRI: magnetic reasonance imaging. 
Table 2. Human plasma lipid mediator (LM) (pg/mL).

\begin{tabular}{|c|c|c|c|c|c|}
\hline & & & HS & MS & $P$ \\
\hline AA bioactive metabolome & Q1 & Q3 & & & \\
\hline $\mathrm{AA}$ & 303 & 259 & $13940 \pm 1942$ & $12480 \pm 811.1$ & 0.4127 \\
\hline LTB4 & 335 & 195 & $32.79 \pm 11.55$ & $63.16 \pm 16.25$ & 0.2958 \\
\hline 20-OH-LTB4 & 351 & 195 & - & - & - \\
\hline 20-COOH-LTB4 & 365 & 195 & - & - & - \\
\hline 5S,12S-diHETE & 335 & 195 & $21.04 \pm 11.04$ & $64.11 \pm 40.07$ & 0.2406 \\
\hline 5S,15S-diHETE & 335 & 115 & $10.78 \pm 5.02$ & $47.30 \pm 14.48$ & 0.1336 \\
\hline PGD2 & 351 & 233 & $1.69 \pm 0.44$ & $8.42 \pm 1.63$ & 0.0211 \\
\hline PGE2 & 351 & 189 & $3.84 \pm 1.22$ & $16.37 \pm 2.68$ & 0.0098 \\
\hline PGF2a & 353 & 193 & $8.55 \pm 3.14$ & $14.74 \pm 1.69$ & 0.0768 \\
\hline TBX2 & 369 & 169 & $63.14 \pm 20.03$ & $209.60 \pm 40.96$ & 0.0289 \\
\hline 5-HETE & 319 & 115 & $199.6 \pm 39.91$ & $345.20 \pm 97.29$ & 0.1588 \\
\hline 12-HETE & 319 & 179 & $657.10 \pm 145.50$ & $1472.00 \pm 180.20$ & 0.0095 \\
\hline 15-HETE & 319 & 219 & $56.98 \pm 14.84$ & $102.70 \pm 11.85$ & 0.0348 \\
\hline LXA4 & 351 & 115 & $0.65 \pm 0.56$ & $1.85 \pm 0.88$ & 0.4077 \\
\hline LXB4 & 351 & 221 & $1.64 \pm 0.44$ & $4.71 \pm 1.66$ & 0.2705 \\
\hline AT-LXA4 & 351 & 115 & $3.56 \pm 1.69$ & $6.57 \pm 1.59$ & 0.2797 \\
\hline AT-LXB4 & 351 & 221 & - & - & - \\
\hline \multicolumn{6}{|l|}{ DHA bioactive metabolome } \\
\hline DHA & 327 & 283 & $25330.00 \pm 3090.00$ & $22690.00 \pm 3133.00$ & 0.6247 \\
\hline RvD1 & 375 & 121 & $0.19 \pm 0.09$ & $0.68 \pm 0.32$ & 0.2735 \\
\hline RvD2 & 375 & 215 & - & - & - \\
\hline RvD3 & 375 & 147 & - & - & - \\
\hline RvD4 & 375 & 101 & - & - & - \\
\hline RvD5 & 359 & 199 & $0.38 \pm 0.15$ & $1.37 \pm 0.43$ & 0.1794 \\
\hline RvD6 & 359 & 101 & - & - & - \\
\hline AT-RvD1 & 375 & 121 & - & - & - \\
\hline AT-RvD3 & 375 & 147 & - & - & - \\
\hline PD1 & 359 & 153 & $0.02 \pm 0.01$ & $0.14 \pm 0.03$ & 0.0325 \\
\hline AT-PD1 & 359 & 153 & - & - & \\
\hline PDX & & & $0.43 \pm 0.15$ & $2.08 \pm 0.65$ & 0.1182 \\
\hline Maresin 1 & 359 & 221 & - & - & - \\
\hline 17-HDHA & 343 & 245 & $71.47 \pm 17.66$ & $114.70 \pm 12.40$ & 0.0588 \\
\hline 14-HDHA & 343 & 205 & $310.20 \pm 91.26$ & $784.90 \pm 130.90$ & 0.0333 \\
\hline 4-HDHA & 359 & 101 & $30.19 \pm 6.94$ & $69.51 \pm 18.37$ & 0.1810 \\
\hline 7-HDHA & 359 & 250 & $5.68 \pm 1.29$ & $7.23 \pm 1.06$ & 0.4185 \\
\hline \multicolumn{6}{|l|}{ EPA bioactive metabolome } \\
\hline $\mathrm{EPA}$ & 301 & 259 & $6605.00 \pm 1296.00$ & $4606.00 \pm 341.80$ & 0.0472 \\
\hline RvEl & 349 & 195 & - & - & - \\
\hline RvE2 & 333 & 253 & - & - & - \\
\hline RvE3 & 333 & 201 & - & - & - \\
\hline 18-HEPE & 317 & 259 & $75.50 \pm 28.23$ & $49.89 \pm 8.10$ & 0.2445 \\
\hline 15-HEPE & 317 & 219 & $18.54 \pm 5.76$ & $17.85 \pm 2.32$ & 0.6215 \\
\hline 12-HEPE & 317 & 179 & $244.70 \pm 66.11$ & $393.70 \pm 49.88$ & 0.0895 \\
\hline 5-HEPE & 317 & 115 & $27.07 \pm 7.79$ & $30.16 \pm 6.90$ & 0.7989 \\
\hline
\end{tabular}

HS: healthy subjects; MS: multiple sclerosis. 

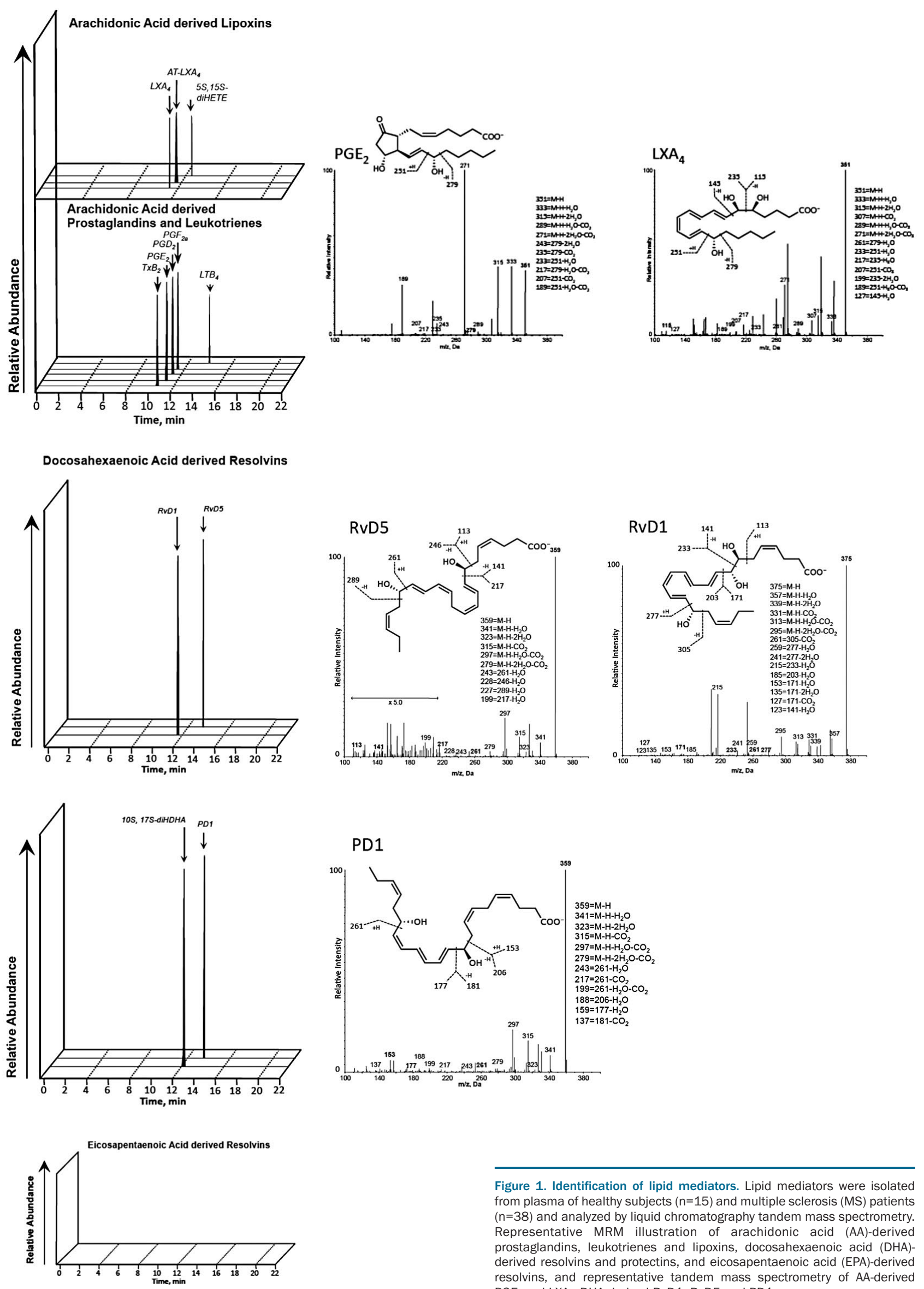

Figure 1. Identification of lipid mediators. Lipid mediators were isolated from plasma of healthy subjects $(n=15)$ and multiple sclerosis (MS) patients $(n=38)$ and analyzed by liquid chromatography tandem mass spectrometry. Representative MRM illustration of arachidonic acid (AA)-derived prostaglandins, leukotrienes and lipoxins, docosahexaenoic acid (DHA)derived resolvins and protectins, and eicosapentaenoic acid (EPA)-derived resolvins, and representative tandem mass spectrometry of AA-derived $\mathrm{PGE}_{2}$ and $\mathrm{LXA}_{4}$, DHA-derived RvD1, RvD5 and PD1. 
A

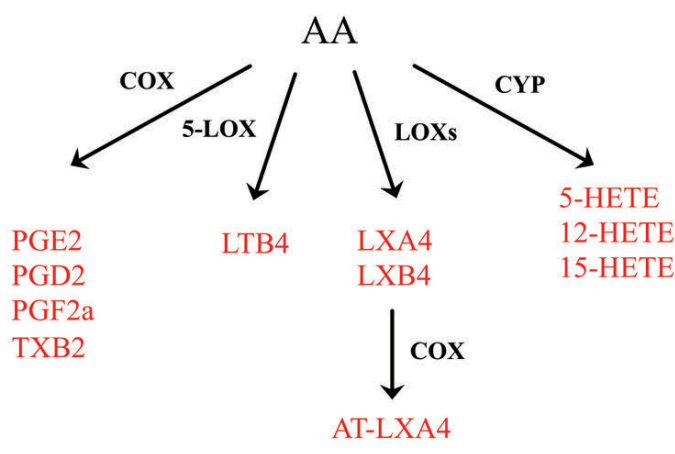

B

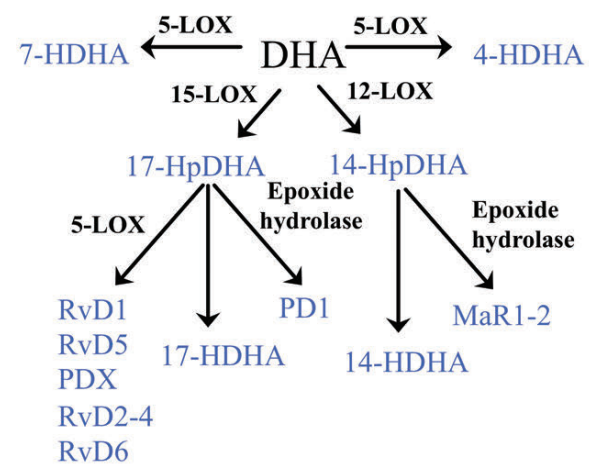

C

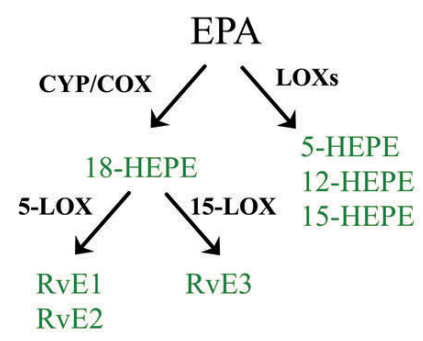

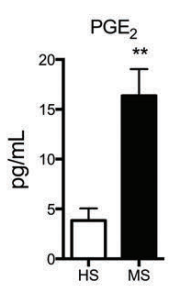
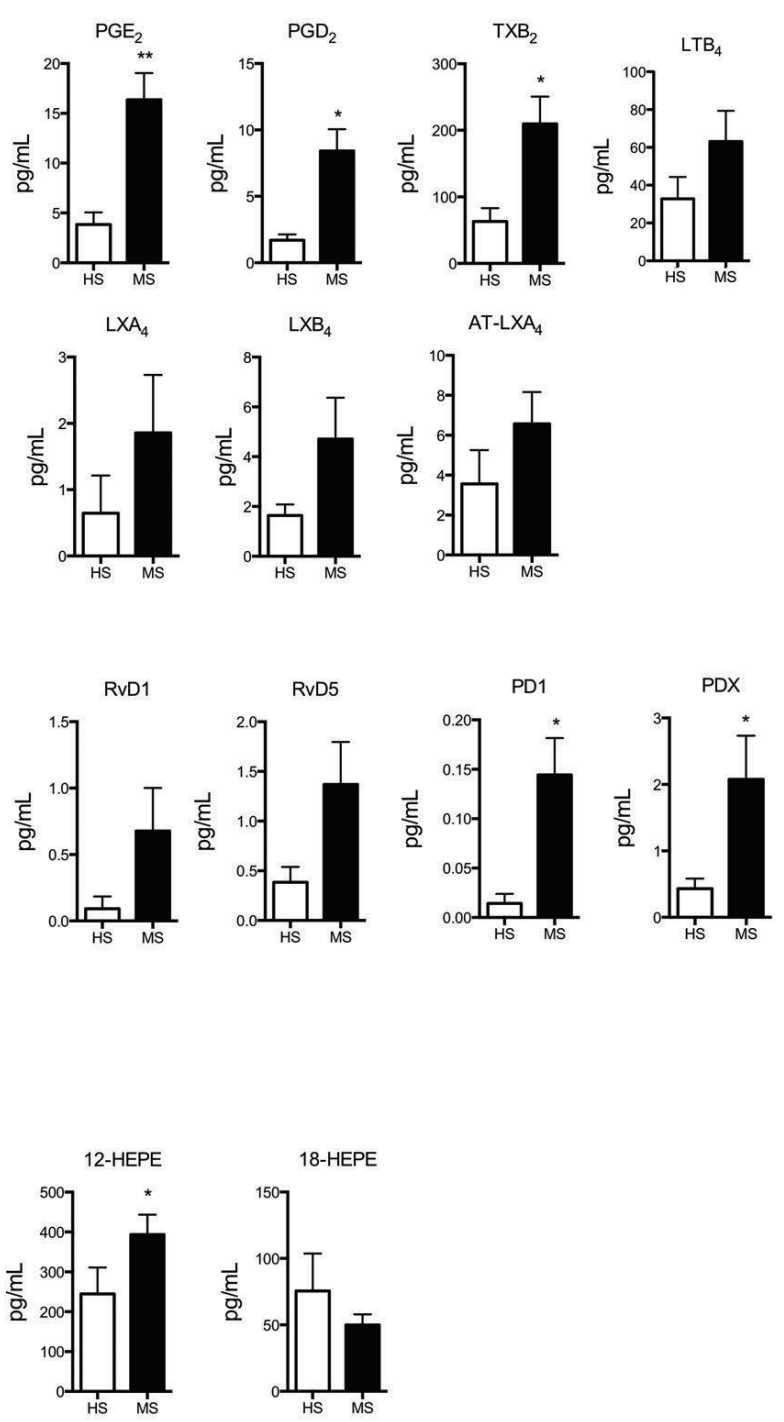

Figure 2. Multiple sclerosis (MS) patients show altered lipid mediators profiles in blood. Lipid mediators (LM) were isolated from plasma of healthy subjects (HS, $n=15)$ and MS patients $(n=38)$ and analyzed by liquid chromatography tandem mass spectrometry. (A) Schematic representation of the arachidonic acid (AA)-derived respective LM biosynthetic pathways and their selected values between HS and MS. (B) Schematic representation of the docosahexaenoic acid (DHA)-derived respective LM biosynthetic pathways and their selected values between HS and MS. (C) Schematic representation of the eicosapentaenoic acid (EPA)-derived respective LM biosynthetic pathways and their selected values between HS and MS. Data are presented as means pg/mL \pm standard error of mean. $* P<0.05$ or $* * P<0.01$ compared to HS, determined by Student $t$-test.

abundance for each gene in respect with beta actin or GAPDH expression.

\section{Electric cell-substrate impedance sensing}

hCMEC/D3 cells were seeded on collagen-coated 96W10idf electric cell-substrate impedance sensing (ECIS) arrays (Ibidi). Trans-endothelial electrical resistance (TEER) of hCMEC/D3 cells was measured at multiple frequencies, ${ }^{21}$ and TNF- $\alpha$ was added as maximum barrier resistance was reached, in the presence or absence of different SPM. Subsequently, TEER was measured over time and finally analyzed. ${ }^{22}$

\section{ELISA}

hCMEC/D3 culture supernatants harvested 24 hours after TNF- $\alpha$ treatment in the presence or absence of SPM were meas- ured for the levels of CCL2/MCP-1 by its commercial ELISA Kit (Invitrogen, the Netherlands) according to the manufacturer's instructions

\section{Transwell migration of monocytes}

In vitro monocyte transendothelial cell migration assay was performed using a collagen 1-coated Transwell system. Briefly, hCMEC/D3 cells were cultured alone or with TNF- $\alpha$ upon which resting or SPM-treated purified human monocytes were added to the transwell filters. ${ }^{23,24}$ Transmigrated cells were determined through flow cytometry.

\section{Statistical analysis}

All data were expressed as means \pm standard error of mean (SEM). Differences between groups were compared using Student 

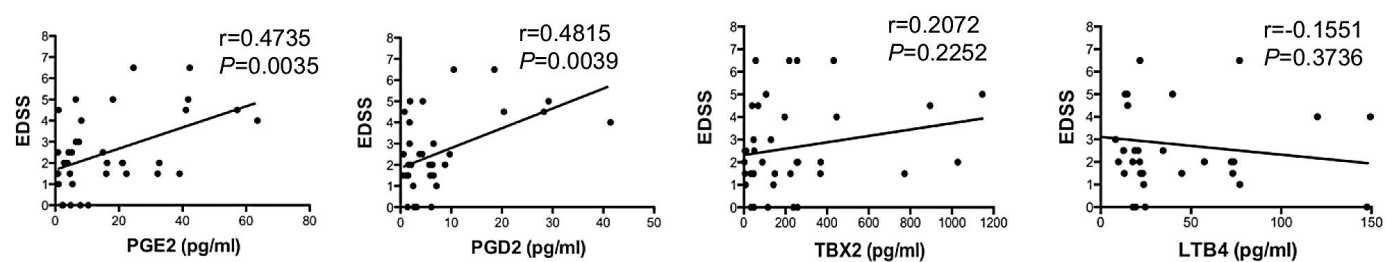

AA

metabolome
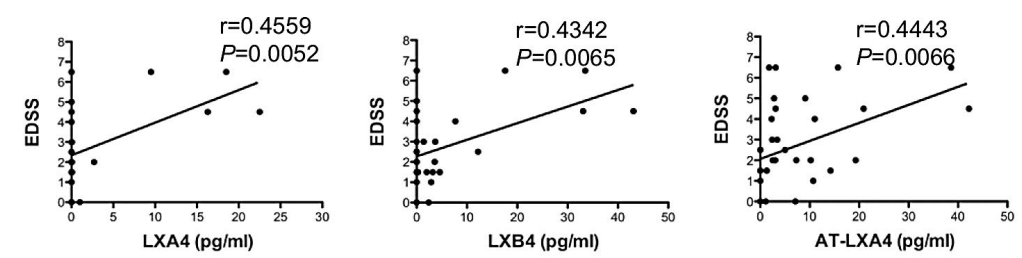

B

DHA metabolome
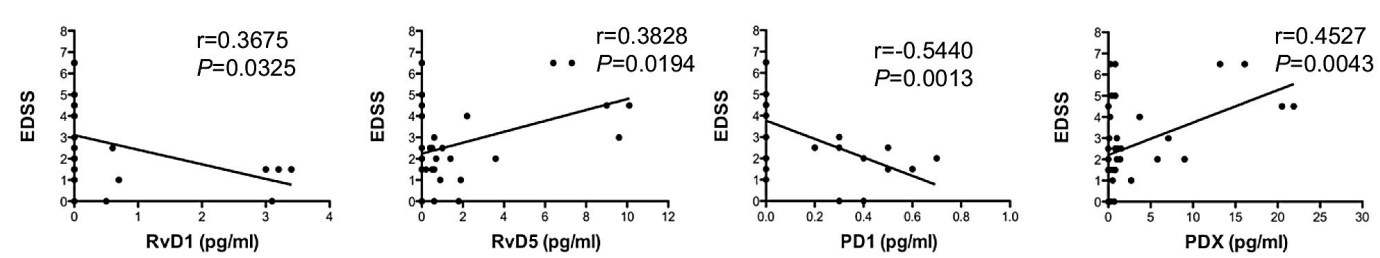

C

EPA

metabolome
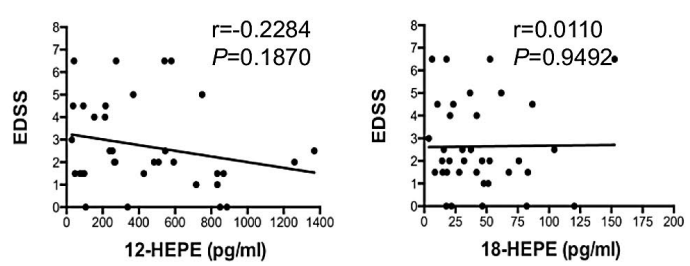

Figure 3. Correlations between Expanded Disability Status Scale (EDSS) scores of patients and lipid mediators. Correlation plots between EDSS values and levels $(\mathrm{pg} / \mathrm{mL})$ of specific lipid mediators of the AA metabolome $(A)$, DHA metabolome $(B)$ and EPA metabolome $(C)$ in the entire cohort of patients with multiple sclerosis. Data were compared by Spearman's rank correlation coefficient $(P<0.05)$.

$t$-test (two groups) or one-way ANOVA (multiple groups) followed by a post hoc Bonferroni test. $P<0.05$ was considered statistically significant.

\section{Results}

\section{Multiple sclerosis patients show altered lipid mediators profiles in the blood}

To address potential differences in LM profiles between MS patients and healthy donors, involving both proinflammatory and specialized pro-resolving lipid mediators (SPM), we performed targeted LM metabololipidomics on human plasma samples using liquid chromatography tandem mass spectrometry (LC-MSMS) in two different cohorts of MS patients, analyzing 42 distinct LM from the endogenous substrates AA, DHA and EPA based on published criteria for each LM (i.e. matching chromatographic retention times (RT), fragmentation patterns, and six characteristic and diagnostic ions) ${ }^{15,16}$ This analysis revealed a pronounced biosynthesis of 27 of these LM in the blood of healthy donors and MS patients (Table 2), and the identification of key LM, including leukotriene $\mathrm{B}_{4}\left(\mathrm{LTB}_{4}\right)$, resolvin (Rv) D1, RvD5 and protectin D1 (Figure 1). Quantitation of LM was performed using signature ion pairs via multiple reaction monitoring (MRM) and revealed marked differences in several LM of each metabolome between MS patients and healthy subjects (Figure 2). In particular, total MS patients showed significantly higher blood levels of many AAderived pro-inflammatory LM, such as prostaglandins (PG) $\mathrm{PGE}_{2}, \mathrm{PGD}_{2}$ (Figure 2A) and hydroxyeicosatetraenoic acids (HETE) 12-HETE and 15-HETE (Online Supplementary Figure S1A), as well as increases, although not significant, in leukotriene $\mathrm{B}_{4}$ and in AA-derived proresolving mediators lipoxins (LX) $\mathrm{A}_{4}$ and $\mathrm{LXB}_{4}$ (Figure 2A). Furthermore, as for LM derived from the DHA metabolome, we observed that MS patients displayed significantly higher levels of pathway markers 17-HDHA and 14-HDHA (Online Supplementary Figure S1B); however, among the ten possible DHA-derived SPM (D-series resolvins, protectins and maresins), only four were detected, e.g. RvD1, RvD5, PD1 and PDX, and these were all generally increased in MS patients, with PD1 and PDX 
A
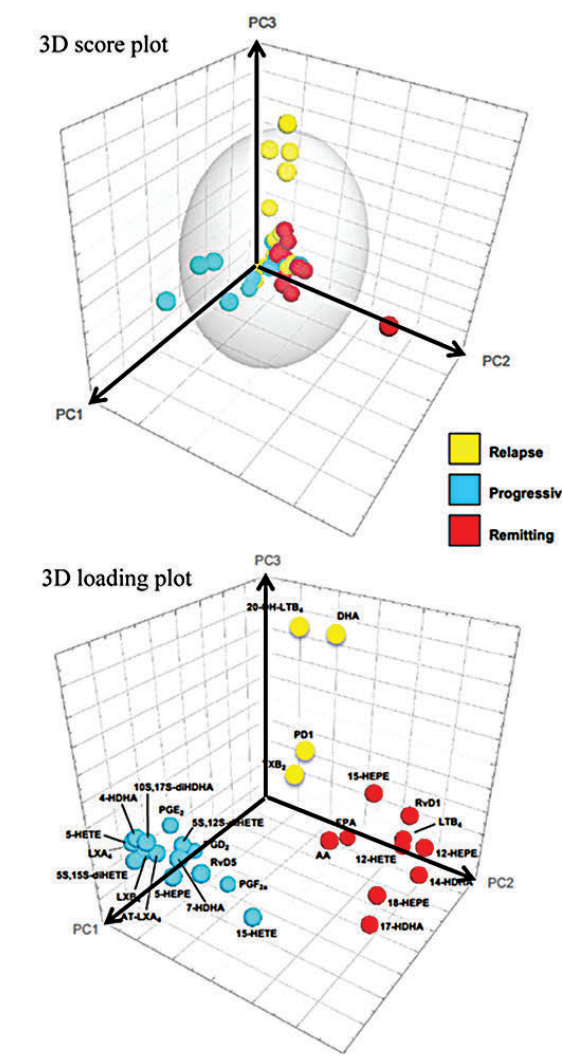
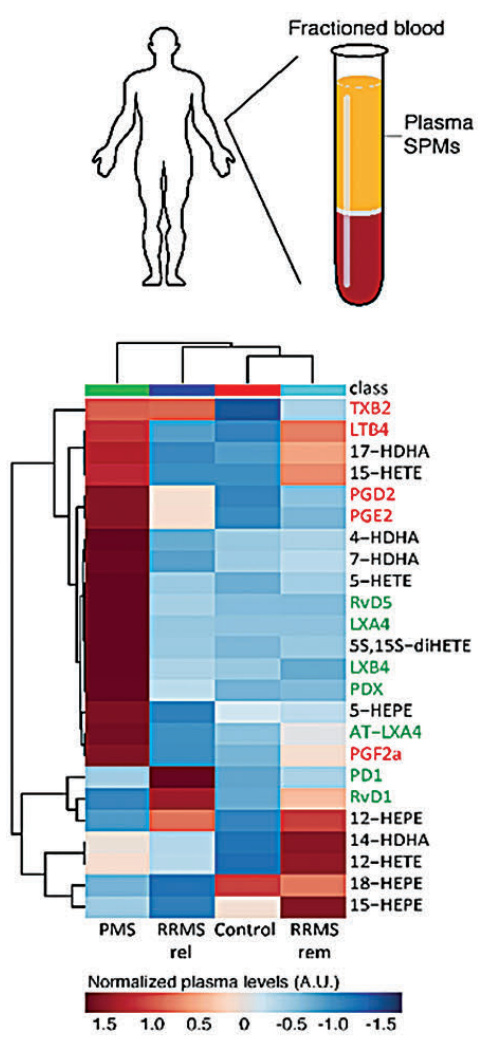

C
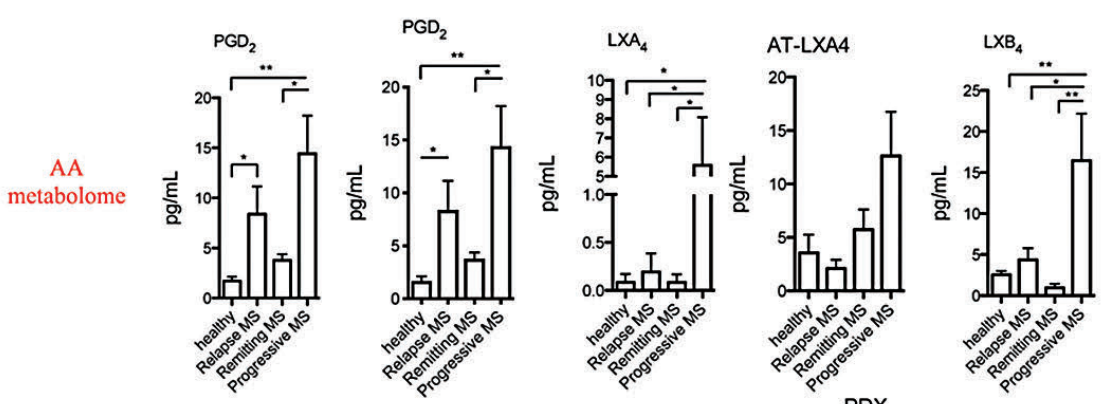

D
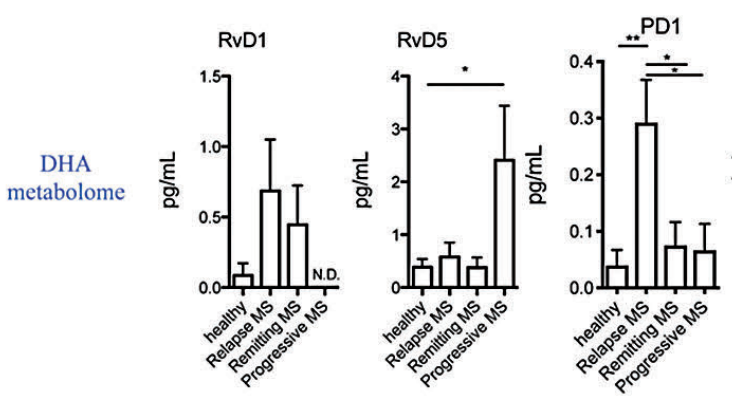

PDX

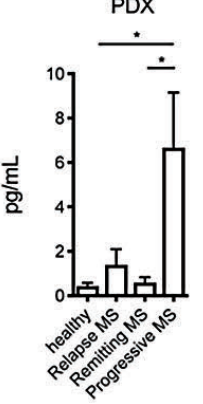

E

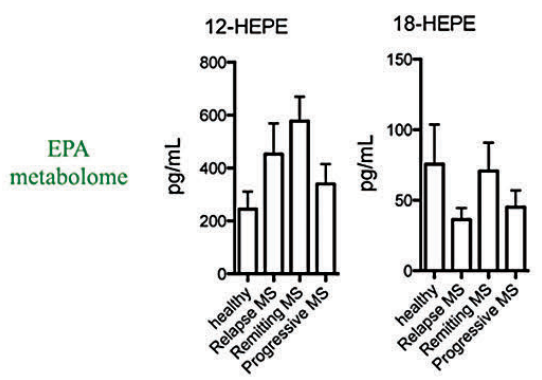

EPA 
A
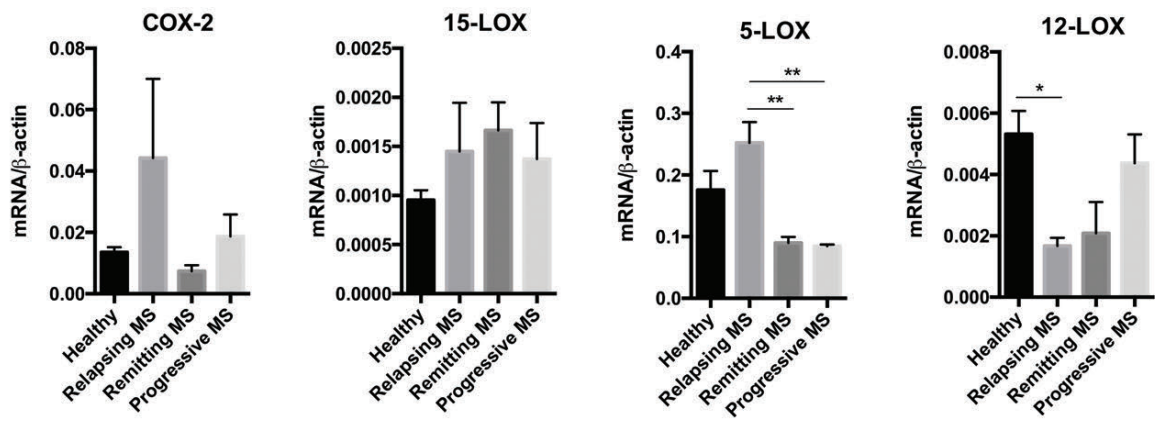

B
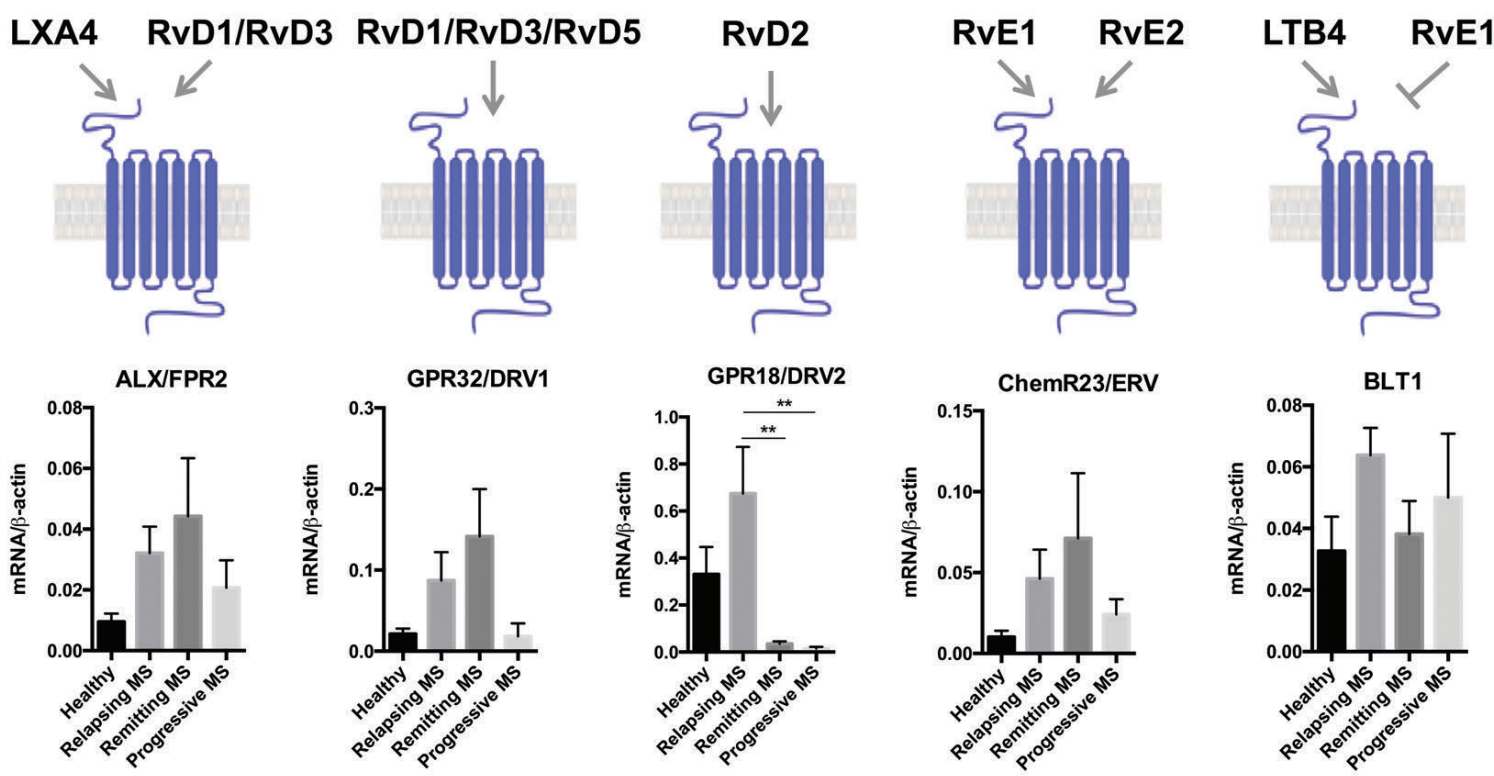

Figure 5. Specialized pro-resolving mediators biosynthetic enzymes and receptors are differentially expressed in multiple sclerosis (MS) patients according to the clinical disease phase. Peripheral blood mononuclear cells (PBMC, $2 \times 10^{6} \mathrm{cells}$ ) from healthy subjects $(n=5)$, relapsing MS ( $n=7$ ), remitting MS ( $=5$ ), and progressive MS $(n=5)$ patients were quantified for their mRNA content by quantitative real-time polymerase chain reaction (qRT-PCR) of lipid mediator biosynthesizing enzymes COX-2, 5-LOX, 12-LOX and 15-LOX (A) and of SPMs receptors ALX/FPR2, GPR32/DRV1, GPR18/DRV2, ChemR23/ERV and BLT1 (B). Data are means \pm standard error of mean of 5-7 independent experiments. ${ }^{*} P<0.05, * * P<0.01$ determined by one-way ANOVA followed by Bonferroni's multiple comparison test.

being significant (Figure 2B). Of note, other DHA-derived SPM, such as RvD2, RvD3, RvD4 RvD6, AT-RvD1, ATRvD3 and maresin (MaR) 1 were undetectable (Table 2). Among the EPA-derived lipid mediators, only 12-HEPE was significantly higher and 18-HEPE was slightly lower in MS patients; levels of E-series resolvins (RvE 1-3) were not identified in these patient samples (Table 2 and Figure 2C). Of interest, the total levels of AA and DHA were almost unchanged between MS patients and healthy subjects, while those of EPA were significantly reduced (Online Supplementary Figure S1). We next observed that all the LM that were increased in MS significantly correlated with disease severity, evaluated as Expanded Disability Status Scale (EDSS) scores, except for $\mathrm{LTB}_{4}$ and EPA-derived 12-HEPE and 5-HEPE (Figure 3). In contrast, RvD1 and PD1 showed a negative correlation, inasmuch as their levels progressively decreased with clinical score (Figure 3B). Interestingly, we did not observe any correlation with the age and gender of patients, whereby levels of SPM were fairly constant in both males and females (data not shown).
Specialized pro-resolving lipid mediators, their biosynthetic enzymes and receptors are differentially expressed in multiple sclerosis patients according to disease phase

Since MS is characterized by different and independent forms of the disease, ${ }^{1-3}$ we next stratified the metabololipidomics analysis according to disease clinical subtype. Using unbiased PCA (Figure 4A), we observed that each form of disease and healthy subjects were associated with distinct LM profiles. Indeed, relapse patients were associated with a cluster characterized by few LM, including $\mathrm{PD} 1$ and $\mathrm{TXB}_{2}$ and remitting patients with a cluster that included several HEPE and HETE, as well as $\mathrm{LTB}_{4}$ and $\mathrm{RvD1}$, while progressive patients gave a cluster that included the most abundant and diversified LM, from pro-inflammatory $\mathrm{PGE}_{2}$ and $\mathrm{PGD}_{2}$ to anti-inflammatory $\mathrm{LXA}_{4}$ and $\mathrm{LXB}_{4}$. In particular, ANOVA analysis showed that many AA-derived pro-inflammatory and pro-resolving mediators, including $\mathrm{PGD}_{2}, \mathrm{PGE}_{2}, \mathrm{LXA}_{4}, \mathrm{LXB}_{4}$ (Figure $4 \mathrm{~B})$ as well as $\mathrm{TXB}_{2}$ (Online Supplementary Figure S2A) fol- 
lowed a similar trend, being generally increased in both relapsing $\mathrm{MS}$ and progressive $\mathrm{MS}$ patients compared to healthy subjects (with $\mathrm{PGD}_{2}$ and $\mathrm{PGE}_{2}$ being also significant for relapsing $\mathrm{MS}$ and $\mathrm{LXA}_{4}$ and $\mathrm{LXB}_{4}$ for progressive $\mathrm{MS}$ ), while showing reduced levels in remitting MS patients compared to clinically active forms. For all of these LM, progressive patients consistently displayed higher levels compared to relapsing patients (Figure 4B and $\mathrm{C}$ ). Of note, levels of their precursor AA moved in the opposite direction (Online Supplementary Figure S2A), suggesting an active metabolic conversion into such LM associated to disease phase. Other key metabolites of AA, including HETE, $\mathrm{PGF}_{2 \mathrm{a}}$ and $\mathrm{LTB}_{4}$, appeared with specific trends of expression, with 5-HETE being mostly found in progressive MS and 12-HETE in remitting MS, while 15-HETE and PGF $_{2 \mathrm{a}}$ steadily increased along disease forms (Online Supplementary Figure S2A). Levels of aspirin-triggered (AT) lipoxins were detected only for AT-LXA which was particularly present in progressive patients (Table 2 and Figure 4B). As for the DHA metabolome, proresolving LM RvD5 and PDX were slightly increased in relapsing $\mathrm{MS}$ and significantly increased in progressive MS, with remitting MS showing similar levels compared to healthy subjects (Figure 4C). Furthermore, RvD1 and PD1 were increased in relapsing MS, with the latter being also significant and showing a reduction in both remitting and progressive patients, while the former being reduced in remitting $M S$ and undetectable in progressive $M S$ (Figure 4C). Of interest, the levels of their precursor DHA, although showing an initial, yet not significant, increase in relapsing $\mathrm{MS}$, were progressively reduced along disease forms (Online Supplementary Figure S2B). Other pathway makers and metabolites of DHA were significantly increased, especially in remitting (14-HDHA and 17HDHA) or progressive (17-HDHA and 4-HDHA) patients (Online Supplementary Figure S2B). As for EPA metabolome, levels of 15-HEPE and 18-HEPE were reduced in relapsing and progressive $\mathrm{MS}$, returning back to control levels in remitting $\mathrm{MS}$, showing a similar trend as their precursor EPA (Figure 4D and Online Supplementary Figure S2C). However, 12-HEPE was particularly high in both relapsing and remitting MS (Figure 4E). Specific LM fingerprints are also shown by volcano plots when comparing every two groups against each other, with SPM like RvD1 and PD1 being reduced along disease progression and others like $\mathrm{LXB}_{4}$ and pro-inflammatory prostaglandins being produced during the active phases of disease, especially in progressive patients (Online Supplementary Figure S3).

Of note, both cohorts of healthy donors and MS patients displayed an almost identical LM profile (Online Supplementary Figure S4), with pro-inflammatory AAderived prostanoids being induced in relapsing and progressive patients and reduced during remission, whereas lipoxins being slightly induced during the relapsing phase and even more during the progressive phase. As for the DHA- and EPA-derived SPM, both cohorts showed an induction of RvD1 and PD1 during the relapsing phase, which were both reduced or even undetected along disease progression, and showed a significant induction of RvD5 in progressive patients. The only SPM that was discordant was PDX, being significantly induced during the relapsing phase in the first cohort of MS patients, whereas in the second cohort, PDX was markedly induced in progressive patients (Online Supplementary Figure S4).

Having observed that each clinical form of MS is char- acterized by differential profiles in the levels of SPM, we next sought to evaluate whether this was associated to contradistinctive capacities to produce them and/or to respond to them. Thus, we further characterized the different forms of MS by investigating the mRNA expression of the main SPM biosynthetic enzymes and their known receptors in peripheral blood leukocytes. While COX-2 and 5-LOX were particularly induced in relapsing $\mathrm{MS}$ to be then strongly reduced in both remitting and progressive patients, 15-LOX was consistently found in all MS forms (Figure 5A). On the contrary, 12-LOX expression was antithetical, inasmuch as its levels were substantially reduced in relapsing and remitting $\mathrm{MS}$ and started to recover in progressive MS patients (Figure 5A). As for SPM receptor expression, ALX/FPR2, DRV1 and ERV all displayed a similar pattern, with their expression levels being induced in relapsing $\mathrm{MS}$, reaching their highest expression in remitting $\mathrm{MS}$, and then exhibiting a reduction in progressive MS (Figure 5B). In addition, while BLT1 receptor expression was induced only in the active phases of the disease, DRV2 was strongly induced in relapsing $\mathrm{MS}$ and markedly reduced in both remitting and progressive patients, to expression levels much lower than healthy subjects (Figure 5B).

\section{Specific specialized pro-resolving mediators attenuate monocyte inflammatory responses in multiple sclerosis patients}

The observed differences of MS patients in producing distinct SPM profiles prompted us to examine whether peripheral blood leukocytes of MS patients were responsive to the immunomodulatory activity of disease-affected SPM. Accordingly, we tested the ability of SPM that showed an initial induction in relapsing MS patients and are subsequently diminished along disease progression (RvD1 and PD1), and SPM that showed a higher expression in relapsing $M S$ patients and no induction in remitting $\mathrm{MS}\left(\mathrm{LXA}_{4}, \mathrm{LXB}_{4}\right)$, to evaluate their potential to affect the activation and cytokine production of activated monocytes obtained from RRMS patients, i.e. in the disease phase where such mediators were initially increased and then decreased. To do so, we analyzed the expression of activation markers and inflammatory cytokines in SPM-treated monocytes that were then challenged with two different viral Toll-like receptors (TLR) agonists: TLR7 and TLR8 (see Online Supplementary Figure S5A for gating strategy). As expected, the simultaneous stimulation of monocytes of relapsing MS patients with selective agonists of viral Toll-like receptors (TLR) 7 and TLR8 induced a strong upregulation of the activation marker CD69 on their cell surface compared to resting monocytes (Figure 6A). Treatment of activated monocytes with $\mathrm{LXA}_{4}, \mathrm{LXB}_{4}, \mathrm{RvD} 1$ or PD1 caused a significant reduction in CD69 surface expression (Figure 6A), indicating an overall ability of these SPM to attenuate the general activation of myeloid cells. More specifically, all these tested SPM were equally able to significantly reduce the intracellular production of several pro-inflammatory cytokines. Indeed, the high levels of TNF- $\alpha$, IL-1 $\beta$, IL-6 and IL-12 production from activated monocytes of relapsing $\mathrm{MS}$ patients were equivalently and significantly reduced by $\mathrm{LXA}_{4}, \mathrm{LXB}_{4}, \mathrm{RvD} 1$ and $\mathrm{PD} 1$, although RvD1 and PD1 showed a higher vigor in reducing IL-6 production (Figure $6 \mathrm{~B}$ and Online Supplementary Figure S5B). The immunomodulatory activity of these SPM was even 
more evident in activated monocytes of healthy donors (Online Supplementary Figure S6A and B) inasmuch as all tested SPM induced an even stronger reduction of CD69 and of all pro-inflammatory cytokines, suggesting that although their pro-resolving actions are equally function$\mathrm{al}$ in health and disease, cells of MS patients are probably less responsive to SPM. Of note, besides reducing proinflammatory cytokines production, $\mathrm{LXA}_{4}, \mathrm{LXB}_{4}, \mathrm{RvD1}$ and PD1 all equally enhanced the production of the typical anti-inflammatory cytokine IL-10 produced from
TLR7/8-activated monocytes (Figure 6C). Interestingly, the SPM-induced effect on cytokine reduction was not observed with monocytes treated with the pro-inflammatory $\mathrm{LM} \mathrm{LTB}_{4}($ Online Supplementary Figure S6C).

\section{Specific specialized pro-resolving mediators counteract blood-brain barrier dysfunction and attenuate monocyte transendothelial migration}

A key pathological feature of $\mathrm{MS}$ is $\mathrm{BBB}$ dysfunction that ultimately leads to monocyte transmigration into the
A

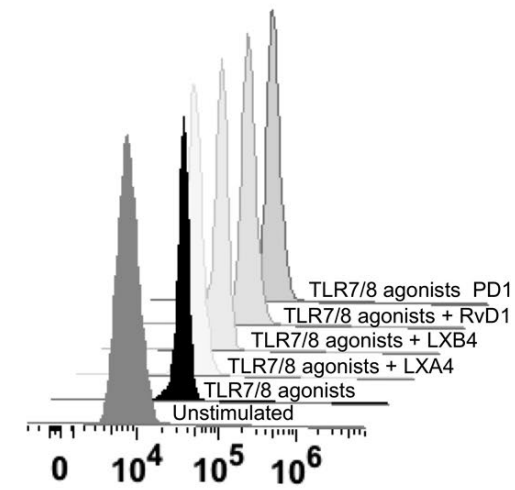

B

IL-6

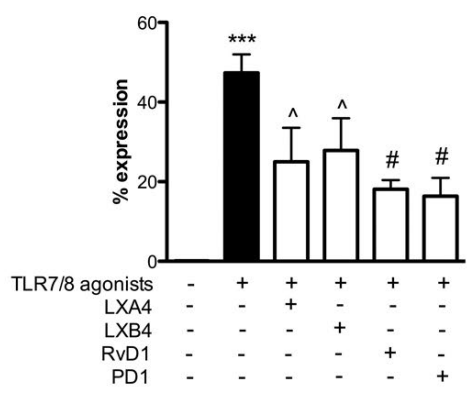

IL-1 $\beta$

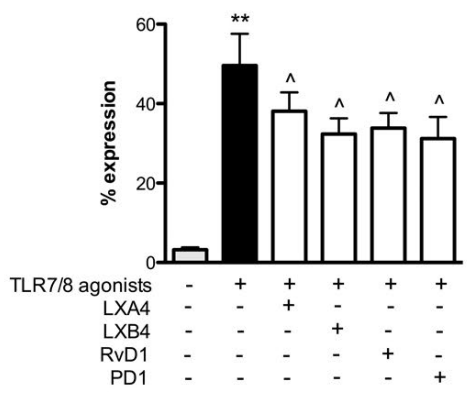

IL-12

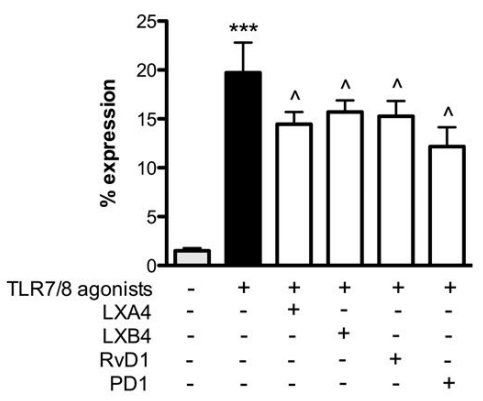

TNF- $\alpha$

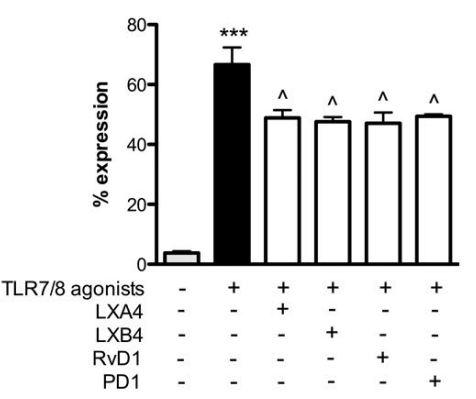

C

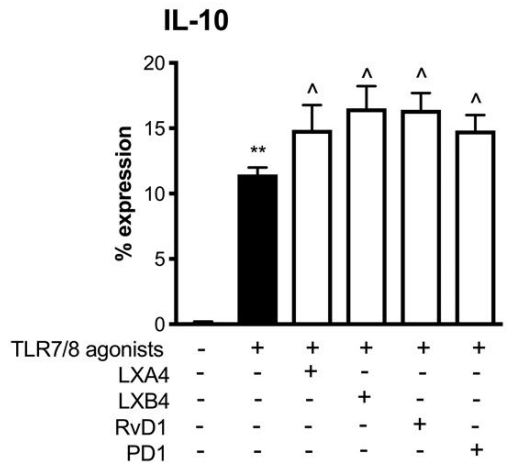

Figure 6. Specialized pro-resolving mediators reduce monocyte activation and cytokine production in multiple sclerosis (MS) patients. Peripheral blood mononuclear cells (PBMC, $2 \times 10^{6}$ cells) from relapsing MS patients $(n=5)$ were left untreated or pre-treated with LXA, LXB, RvD1 or PD1 for 30 minutes. Cells were then stimulated with Imiquimod (Toll-like receptor 7 agonist) and ssRNA40 (Toll-like receptor 8 agonist) for five hours in absence or presence of Brefeldin A, stained at the cell surface and intracellularly, and analyzed by flow cytometry by gating on CD14+ monocytes. (A) Surface expression of CD69 positive monocytes. Data are shown as representative flow cytometry histograms and as means of fluorescence intensity (MFI) \pm standard error of mean of five independent experiments. $* * P<0.01$ compared to control cells and $\wedge P<0.05$ compared to TLR7/TLR8 agonists, determined by one-way ANOVA followed by Bonferroni's multiple comparison test. (B) Cytofluorimetric plots and percentages of intracellular pro-inflammatory cytokine production (IL-6, IL-12, IL1- $\beta$ and TNF- $\alpha$ ) from CD14 ${ }^{+}$monocytes. Data are presented as means \pm standard error of mean (SEM) of five independent experiments. $* * P<0.01$ and $* * * P<0.001$ compared to control cells, $\wedge P<0.05$ and $\# P<0.001$ compared to TLR7/TLR8 agonists, determined by one-way ANOVA followed by Bonferroni's multiple comparison test. (C) Cytofluorimetric plots and percentages of intracellular IL-10 production from CD14 ${ }^{+}$monocytes. Data are presented as means \pm SEM of four independent experiments. $* * P<0.01, \wedge P<0.05$ and $\# P<0.001$ compared to TLR7/TLR8 agonists, determined by one-way ANOVA followed by Bonferroni's multiple comparison test. 
A

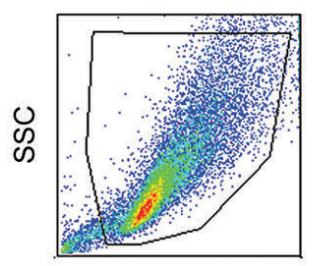

FSC
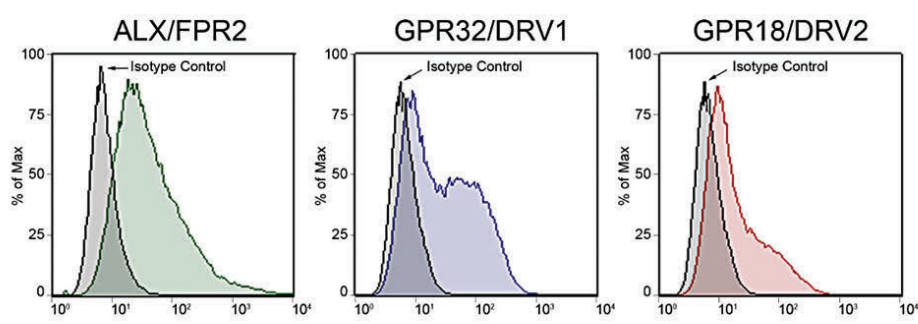

B

Receptor expressions

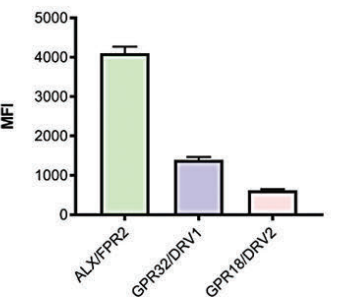

C

ALX/FPR2

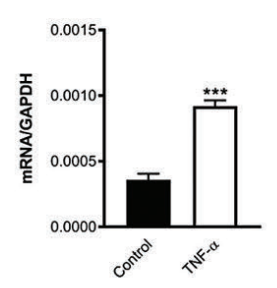

E

TEER 24 hours

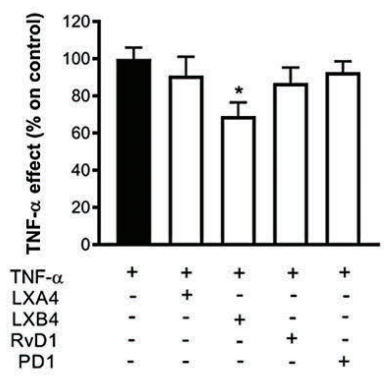

$\mathrm{F}$

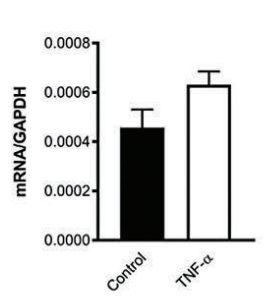

GPR18/DVR2

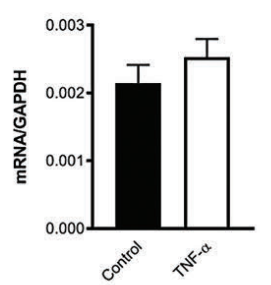

TEER 48 hours

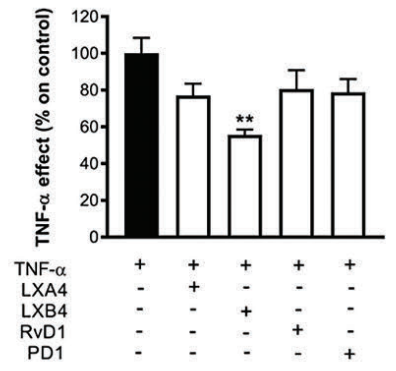

G ICAM-1

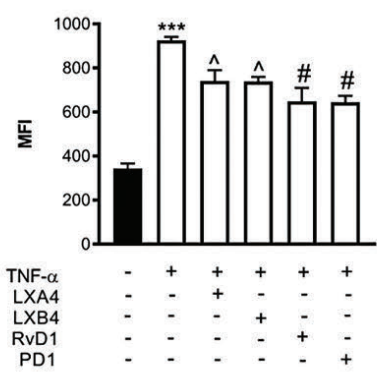

D

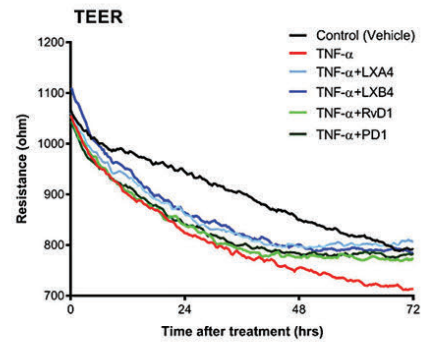

TEER 72 hours

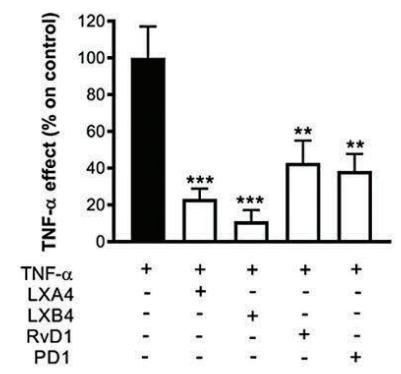

H

CCL2

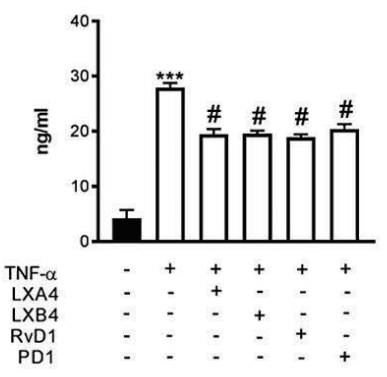

Figure 7. Specialized pro-resolving mediators (SPM) improve blood brain barrier (BBB) function and reduce monocyte transmigration and activation. (A) Representative scatter plots of forward scatter versus side scatter from human brain endothelial cells (BEC) and representative overlays histogram plot gated on live cells for GPR18/DRV2, GPR32/DRV1 and ALX/FPR2 surface expression. (B) Quantification of surface expression. Data are means \pm standard error of mean (SEM) of four independent experiments. (C) SPM receptors mRNA content in resting or TNF- $\alpha$-activated BEC. Data are means \pm SEM of three independent experiments. Statistical analysis was carried out using Student $t$-test. $* * * P<0.001$. (D and E) The functional effect of TNF- $\alpha(5 \mathrm{ng} / \mathrm{mL})$ in the presence or absence of $L X A_{4}$, LXB , $_{4}$ RvD1 or PD1 on BBB function was assessed by measuring the trans-endothelial electrical resistance (TEER) of BEC. Confluent BEC monolayer was treated as described and TEER was measured over time. Data are shown as representative TEER curves of three independent experiments. Graphs showing the TNF- $\alpha$ effect at selected time-points, plotted as \% TNF- $\alpha$ effect of control BEC \pm SEM of three independent experiments. Statistical analysis was carried out using Student $t$-test. $* P<0.05, * * P<0.01, * * * P<0.001$. (F-H) Confluent BEC were stimulated for 24 hours with TNF- $\alpha$ in the presence or absence of LXA, LXB, RvD1 or PD1. Human monocytes $\left(1 \times 10^{5}\right.$ cells/well) were left untreated or treated with $\mathrm{LXA}_{4}, \mathrm{LXB}_{4}$, RvD1 or PD1 prior plated on BEC. Cells were incubated for eight hours before harvesting the transmigrated cells. (F) Percentage of monocyte transmigration evaluated by flow cytometry. Data are shown as means \pm SEM of three independent experiments. $* * * P<0.001$ compared to control cells and $\# P<0.001$ compared to TNF- $\alpha$ stimulated cells, determined by one-way ANOVA followed by Bonferroni's multiple comparison test. ICAM-1 expression by flow cytometry $(G)$ and $C C L 2$ secretion was measured by ELISA (H). Data are means $\pm S E M$ of three independent experiments. $* * * P<0.001$ compared to control cells, $\wedge P<0.05$ and $\# P<0.001$ compared to TNF- $\alpha$ stimulated cells, determined by one-way ANOVA followed by Bonferroni's multiple comparison test. 
CNS by crossing the inflamed and disrupted BBB. Therefore, we addressed the question as to whether the differentially expressed SPM were able to counteract inflammation-induced $\mathrm{BBB}$ dysfunction, by using human brain endothelial cells (BEC) as a BBB model. We first assessed if these cells responded to SPM by looking at the expression pattern of specific SPM receptors. We found that BEC mainly express ALX/FPR2 and to a lesser extent GPR18/DVR2 and GPR32/DVR1 (Figure 7A and B). Interestingly, such marked ALX/FPR2 expression was even more evident when BEC were stimulated with TNF- $\alpha$, inasmuch as inflamed cells underwent a significant upregulation of ALX/FPR2 mRNA, while they only showed a slight increase in GPR18/DVR2 and GPR32/DVR1 expression (Figure 7C). Next, we assessed whether $\mathrm{LXA}_{4}, \mathrm{LXB}_{4}, \mathrm{RvD1}$ and PD1 were capable of counteracting inflammation-induced $\mathrm{BBB}$ dysfunction by measuring TEER in real-time. We found that these SPM were able to rescue the TNF- $\alpha$-mediated decrease in TEER in a time-dependent manner, at both a $10 \mathrm{nM}$ (Figure 7D and E) and $100 \mathrm{nM}$ concentration (Online Supplementary Figure S7), with $\mathrm{LXB}_{4}$ starting to have a significant effect as early as at 24 hours (h) (at $10 \mathrm{nM}$ but not at $100 \mathrm{nM}$ ) and with all SPM significantly rescuing TEER after $72 \mathrm{~h}$ (at both concentrations). Of note, no dosedependency in SPM potency in rescuing TEER was observed, except for $100 \mathrm{nM} \mathrm{LXA}_{4}$ that showed a significant impact at $48 \mathrm{~h}$. In view of these results, we next sought to investigate the SPM effect on monocyte transendothelial migration by using this human $\mathrm{BBB}$ model. Treatment with $\mathrm{LXA}_{4}, \mathrm{LXB}_{4}, \mathrm{RvD} 1$ or PD1 significantly inhibited the migration of monocytes across $\mathrm{BEC}$ (Figure 7F) and this action was associated with a significant reduction in the expression of endothelial adhesion molecule ICAM-1 and chemokine CCL2 (Figure 7G and $\mathrm{H})$, thereby accounting for a potent anti-inflammatory action of the pro-resolving LM in preventing inflammation-induced BBB dysfunction.

\section{Discussion}

This study provides an unprecedented comprehensive overview of the LM signature in plasma from MS patients with different clinical forms of the disease compared to healthy controls. Targeted LM metabololipidomics using LC-MS-MS with subsequent analyses revealed that relapsing $\mathrm{MS}$ patients display most of the AA-derived prostaglandins (i.e. $\mathrm{PGD}_{2}$ and $\mathrm{PGE}_{2}$ ) as well as of DHAderived SPM like PD1 and RvD1 (and partly PDX), which were all reduced in remitting MS patients. In addition, progressive MS patients not only were characterized by the co-presence of all pro-inflammatory mediators (all of them even in higher levels than relapsing patients) but also on the appearance of some AA-derived or DHA-derived SPM, such as $\mathrm{LXA}_{4}$ and $\mathrm{LXB}_{4}, \mathrm{RvD} 5$ and PDX. Of note, the levels of PD1 and RvD1 were significantly reduced or even undetected along disease progression.

In line with the general concepts that bioactive LM undergo temporal and spatial production during inflammation, that SPM appear at the peak of acute inflammation in order to later reduce inflammation by activating endogenous resolution programs, ${ }^{7,10}$ and that chronic inflammation may result from failed resolution mechanisms, ${ }^{12,13}$ our results display that during the acute phase of the disease (relapsing form) there is an imbalance between pro-inflammatory and pro-resolving LM, in favor of the former, and an insufficient or lack of expression of many key SPM (including E-resolvins, maresins and the rest of D-resolvins), which may in turn affect the outcome of remission and thereby, in theory, even lead to disease progression, although further studies are needed to fully clarify this. Indeed, over $80 \%$ of individuals with MS initially develop a clinical pattern with periodic relapses that reflect acute inflammation in the CNS and myelin disruption as well as induced activation of innate immune cells and pro-inflammatory mediators in peripheral blood, followed by continuous remissions during which selfremyelination occurs and symptoms decrease or temporarily disappear. ${ }^{6,25}$ Repeated relapses and remissions lead to less and less effective remyelination, appearance of scar-like plaques (scleroses) and thus after 10-20 years, patients might evolve into a progressive form of the disease, characterized by an irreversible disruption of peripheral and central immune tolerance, neurodegeneration, and permanent cortical and subcortical gray matter atrophy. ${ }^{26}$ More than a dozen disease-modifying (and mostly anti-inflammatory) agents are available to reduce the frequency of transient episodes of neurological disability and limit the accumulation of CNS lesions, but these systemically applied agents not only are exclusive for RR-MS patients and not for progressive patients, but also result in severe side-effects, and none of these prevents or reverses the neurological deterioration. ${ }^{27}$ Therefore, we set out to investigate whether impairments of endogenous processes to resolve inflammation correlate with MS progression to ultimately provide tools to either slow down inflammatory activation and simultaneously promote neuroprotection or prevent disease progression. The primary objective of our study was to identify pro-inflammatory and proresolving $\mathrm{LM}$ and validate their structures in peripheral blood of MS patients and healthy controls, and secondly, to correlate levels with clinical outcomes. To this aim, targeted metabololipidomics allowed us to reveal the full spectrum of LM in plasma samples of healthy donors and of MS patients with different clinical disease forms. We found that acute MS patients are able to produce only very few SPM (i.e. lipoxins, RvD1 and PD1). This is suggestive of a defective resolution program during MS that could not only result in a partial recovery, but could also eventually increase the probability of evolving into the progressive form, as substantiated by an inverse correlation of such SPM with clinical severity. Our observed presence of high levels of few SPM (LXA $\mathrm{LXB}_{4}, \mathrm{RvD5}$ and $\mathrm{PDX}$ ) in progressive $\mathrm{MS}$, which instead positively correlate with clinical severity, is indicative of a last, but ineffective, attempt of the body to respond to an even higher inflammatory status, where all pro-inflammatory LM are consistently produced in high amounts and are also associated to disease severity. This is particularly relevant for $\mathrm{LXA}_{4}$ and $\mathrm{LXB}_{4}$ that are metabolically derived from arachidonic acid, and that are the actual initiators of the metabolic switch from the omega-6 pro-inflammatory eicosanoids to the omega-3 SPM. Indeed, MS patients attempt to induce a compensatory boost of these two lipoxins in order to promote the subsequent production of all SPM, which is reflected only in an increased production of RvD5 and PDX (whose potency is much lower than its stereoisomer PD1) and not by an induction of all other SPM. Of note, typical SPM that are usually produced later 
in the inflammatory process, and that appear also during chronic stages, namely RvD3 and RvD4, are undetected in all MS phases, further suggesting that, although progressive patients endeavor in a last attempt to boost a lipoxinmediated metabolic switch towards SPM, this is not followed by actual SPM production.

Our findings are in line with the only other study that analyzed a few of such LM in MS, whereby RvD1 and PD1 were induced in highly active MS patients. ${ }^{28}$ However, this study, which was performed on cerebrospinal fluid samples, not only analyzed a smaller cohort of patients and did not take into consideration healthy subjects, but was able to detect only one-third of the LM that have been measured here. Furthermore, and most importantly, our metabololipidomics analysis was performed on three clinically distinct MS forms, which included not only $\mathrm{MS}$ with active relapse phases, but also patients with clear signs of remission or progression, allowing us to have a complete overview of a vast array of LM and observe how they vary along disease phases and during progression.

The recent evidence that several chronic inflammatory diseases are associated with altered SPM metabolism also supports our findings. Indeed, decreased production of lipoxins and resolvins (especially RvD1) have been linked to the pathogenesis of chronic obstructive pulmonary disease, ${ }^{29}$ type- 2 diabetes and obesity, ${ }^{30,31}$ inflammatory bowel disease, ${ }^{32,33}$ and rheumatoid arthritis. ${ }^{34}$ In addition, an imbalance between pro-inflammatory leukotrienes and pro-resolving SPM was observed in atherosclerosis. ${ }^{35} \mathrm{Of}$ note, the notion that also neuroinflammatory and neurodegenerative diseases might be linked to a dysfunctional resolution of inflammation has very recently been put forward, and an impaired pro-resolution pathway, involving both specific SPM (i.e. $\mathrm{LXA}_{4}$ and RvD1) and their receptors was found in post-mortem brain tissues of patients with Alzheimer disease, ${ }^{36,37}$ where clinical trials with DHA show a reduced peripheral inflammation associated with increases in specific SPM. ${ }^{38}$ Accordingly, our observed significant and progressive reduction of DHA during MS, reaching very low levels in progressive patients, once again support a defect in producing its SPM derivatives. In line with this, Holmann et al. described deficiencies in polyunsaturated fatty acid (PUFA) and subsequent replacement by non-essential fatty acids in $\mathrm{MS}^{39}$ Along these lines, untargeted metabolomics analysis of plasma samples derived from mice with experimental autoimmune encephalomyelitis (EAE), the most commonly used animal model for MS, revealed similar profound alterations in the omega-3 and omega-6 PUFA pathways, with several metabolites of PUFA being significantly lower in EAE mice including RvD1. ${ }^{40}$ Importantly, RvD1 supplementation ameliorated clinical signs of EAE, illustrating in vivo efficacy of SP during neuro-inflammation. ${ }^{40}$ Epidemiological studies suggest that in particular omega-3 PUFA supplementation is linked with improved clinical outcomes in patients with MS. ${ }^{27,41,42}$ However, although the levels of AA and DHA could be restored by supplementation in MS patients, the efficacy of PUFA supplementation remains to be established.

Next, we further investigated the profile of peripheral blood leukocytes, and our analysis revealed distinctive expressions patterns of SPM biosynthetic enzymes and receptors in each clinical form of MS. Relapsing MS patients showed increased expression of COX-2 and 5-
LOX as well as of all five identified receptors (ALX/FPR2, GPR2/DRV1, GPR18/DRV2, ChemR23/ERV and BLT1). Interestingly, 12-LOX, which is responsible for maresins production, was consistently lower in all MS phases compared to healthy donors. Furthermore, the expression of SPM enzymes and receptors decreased along disease progression, with the exception of 15-LOX (that remained constant in all MS phases) and of ALX/FPR2, GPR32/DRV1, and ChemR23/ERV that further increased only during remission, thus suggesting their possible involvement in promoting pro-resolution programs and neuroprotection, but subsequently dropped in progressive MS patients.

It is worth mentioning that the levels of SPM observed in the different disease phases might also be a consequence of a differential utilization and/or degradation, as well as a different expression, of their target receptors. Indeed, progressive patients bear the lowest amount of all SPM target receptors, yet they continue to express high levels of the proinflammatory BLT1 receptor, whose action is only blocked by E-series resolvins that are never to be found in all MS phases.

Although many types of leukocytes are involved in disease progression, activated monocytes are believed to be one of the first to arrive to the brain and initiate inflammation. ${ }^{43}$ In MS, the majority of monocytes display a classical inflammatory phenotype and are hyperactive. ${ }^{44}$ Here we found that monocytes isolated from RR-MS patients not only displayed a more activated and pro-inflammatory status (since their expression of CD69 and cytokines were, indeed, much higher than monocytes of healthy subjects), but also that specific SPM significantly inhibited such inflammatory responses in both healthy monocytes and those of RR-MS patients. However, the ability of SPM to modulate the inflammatory response of these peripheral cells was more evident in cells of healthy subjects, suggesting that, despite expressing comparable levels of proresolving receptors, MS patient-derived monocytes are less susceptible to SPM. These findings confirm and extend earlier reports in which such SPM were shown to reduce the inflammatory profile of human monocytes upon a pro-inflammatory stimulus. ${ }^{45-47}$ Of note, such SPMinduced effect is of crucial importance in preventing priming and activation of autoreactive $T$ cells (especially Th1 and Th17 cells) and natural killer cells, whose pathogenicity are strictly dependent on monocyte-derived cytokines.

Of note, the onset of MS starts when activated and autoreactive peripheral immune cells cross the BBB and start to damage myelin. In this process, BBB endothelial cells are key regulators of the neuroinflammatory response, inasmuch as when inflamed they lead to BBB disruption, upregulation of several adhesion molecules and production of chemokines, ultimately favoring leukocyte transmigration and subsequent MS lesion development. ${ }^{48}$ However, BBB endothelial cells also play an important role during the resolution phase of inflammation via the secretion of pro- and anti-inflammatory mediators that co-ordinate both leukocyte traffic and barrier function. ${ }^{49}$ In this context, despite the fact that a great number of studies have shown the anti-inflammatory and proresolving effect of SPM on various cell types of the immune system, their potential impact on inflamed $\mathrm{BBB}$ has never been reported. Our results show for the first time, not only that BBB endothelial cells express several pro-resolving receptors, which are increased upon inflam- 
mation, but also that specific SPM (LXA $\mathrm{LXB}_{4}, \mathrm{RvD} 1$ and $\mathrm{PD} 1)$ can prevent inflammation-induced $\mathrm{BBB}$ dysfunction, and reduce monocyte transmigration, as well as expression of ICAM-1 adhesion molecule and production of CCL2 chemokine. Our results confirm and extend previous findings in which SPM have been shown to positively regulate endothelial barrier functions through different mechanisms of action. Indeed, it has been shown that $\mathrm{LXA}_{4}$ and RvD1 were able to protect LPS-induced barrier integrity and function via suppression of reactive oxygen species (ROS) production, ${ }^{50}$ inhibition of the NF- $\mathrm{KB}$ pathway $^{51}$ or induction of the antioxidant protein Nrf2..$^{52}$ Furthermore, SPM like (AT)-LXA, RvD1, RvD2, and MaR1 were reported to reduce monocyte/macrophage infiltration and chemotaxis both in vitro and in vivo, 5,54 with RvD1 also being able to induce a switch to the antiinflammatory M2 phenotype on monocyte-derived brain macrophages in the murine model of MS. ${ }^{40}$ Although several studies report the anti-inflammatory role of different SPM (LXA 4 in particular) on vascular endothelial cells or monocytes/macrophages, in terms of reduction of ICAM1 expression ${ }^{5,56}$ and CCL2 production, ${ }^{57}$ we are the first to reveal potent SPM effects on the BBB, therefore providing novel tools to counteract inflammation-induced BBB dysfunction.

In conclusion, we provide here a comprehensive profiling of the LM signature in plasma from MS patients with different clinical forms of the disease compared to healthy controls. Importantly, our data indicate that key SPM are lacking at different disease stages, which not only indicates a failed resolution response in these individuals, but may also provide an explanation as to why the disease progresses. It may also hint at novel therapeutic strategies aimed at boosting their endogenous production or at activating their target receptors. At a functional level, we here show that $\mathrm{LXA}_{4}, \mathrm{LXB}_{4}, \mathrm{RvD} 1$ and PD1 significantly reduce the inflammatory profile of MS-patient-derived monocytes and potently inhibit inflammation-induced BBB dysfunction and monocyte-BBB traversal, which are key pathological hallmarks of MS lesion development. Although further investigations are needed to verify whether SPM impairment is also associated to demyelination and behavior/motor functions in MS patients, this study highlights the potential to use SPM as novel blood biomarkers for MS diagnosis and provides novel tools to ultimately limit MS pathogenesis at several clinical disease stages.

\section{Funding}

This study was supported by the Italian Foundation of Multiple Sclerosis (FISM grant 2017/R/08 to VC) and Ministry of Health, Progetto Giovani Ricercatori (GR-2016-02362380 to VC), Nauta Fonds and VUmc MS Center Amsterdam (to GK), Dutch MS Research Foundation (14-878MS to GK), by the European Union's Seventh Framework Program FP7 under Grant agreement 607962 (nEUROinflammation) (to CDT) and studies in the CNS laboratories were supported by NIH/NIGMS (Grant P01GM095467). GK was supported by an IBRO Research Fellowship.

\section{References}

1. Noseworthy JH, Lucchinetti C, Rodriguez M, Weinshenker BG. Multiple sclerosis. N Engl J Med. 2000;343:938-952.

2. Dendrou CA, Fugger L, Friese MA. Immunopathology of multiple sclerosis. Nat Rev Immunol 2015;15:545-558.

3. Compston A, Coles A. Multiple sclerosis. Lancet. 2008;372(9648):1502-1517.

4. Dutta R, Trapp BD. Mechanisms of neuronal dysfunction and degeneration in multiple sclerosis. Prog Neurobiol. 2011; 93(1):1-12.

5. Calabrese M, Magliozzi R, Ciccarelli $O$, Geurts JJ, Reynolds R, Martin R. Exploring the origins of grey matter damage in multiple sclerosis. Nat Rev Neurosci. 2015; 16(3):147-58

6. Mahad DH, Trapp BD, Lassmann $\mathrm{H}$. Pathological mechanisms in progressive multiple sclerosis. Lancet Neurol. 2015; 14(2):183-193.

7. Serhan CN. Pro-resolving lipid mediators are leads for resolution physiology. Nature. 2014;510(7503):92-101.

8. Chiang N, Serhan CN. Structural elucidation and physiologic functions of specialized pro-resolving mediators and their receptors. Mol Aspects Med. 2017;58:114129.

9. Chiurchiù V, Leuti A, Maccarrone M. Bioactive lipids and chronic inflammation: managing the fire within. Front Immunol. 2018;9:38.

10. Leuti A, Maccarrone M, Chiurchiù V. Proresolving Lipid Mediators: Endogenous Modulators of Oxidative Stress. Oxid Med Cell Longev. 2019;2019:8107265.
11. Chiurchiù V, Leuti A, Dalli J et al. Proresolving lipid mediators resolvin D1, resolvin D2, and maresin 1 are critical in modulating $\mathrm{T}$ cell responses. Sci Transl Med. 2016;8(353):353ra111.

12. Nathan C, Ding A. Nonresolving inflammation. Cell (2010);140:871-882

13. Krashia P, Cordella A, Nobili A, La Barbera L, Federici M, Leuti A, Campanelli F, Natale G, Marino G, Calabrese V, Vedele F, Ghiglieri V, Picconi B, Di Lazzaro G, Schirinzi T, Sancesario G, Casadei N, Riess O, Bernardini S, Pisani A, Calabresi P, Viscomi MT, Serhan CN, Chiurchiù V, D'Amelio M, Mercuri NB. Blunting neuroinflammation with resolvin D1 prevents early pathology in a rat model of Parkinson's disease. Nat Commun. 2019; 10(1):3945.

14. Lopes Pinheiro MA, Kooij G, Mizee MR et al. Immune cell trafficking across the barriers of the central nervous system in multiple sclerosis and stroke. Biochim Biophys Acta. 2016;1862(3):461-471.

15. English JT, Norris PC, Hodges RR, Dartt DA, Serhan CN. Identification and profiling of specialized pro-resolving Mediators in human tears by lipid mediator metabolomics. Prostaglandins Leukot Essent Fatty Acids. 2017;117:17-27.

16. Werz O, Gerstmeier J, Libreros $S$ et al. Human macrophages differentially produce specific resolvin or leukotriene signals that depend on bacterial pathogenicity. Nat Commun. 2018;9(1):59.

17. Chiurchiù V, Leuti $\mathrm{A}$, Cencioni MT et al. Modulation of monocytes by bioactive lipid anandamide in multiple sclerosis involves distinct Toll-like receptors.
Pharmacol Res. 2016;113(Pt A):313-319.

18. Hong S, Tian H, Lu Y et al. Neuroprotectin/protectin D1: endogenous biosynthesis and actions on diabetic macrophages in promoting wound healing and innervation impaired by diabetes. Am J Physiol Cell Physiol. 2014;307(11):C1058C1067.

19. Bonnans C, Vachier I, Chavis C, Godard P, Bousquet J, Chanez P. Lipoxins are potential endogenous antiinflammatory mediators in asthma. Am J Respir Crit Care Med. 2002;165(11):1531-1535.

20. Weksler B, Romero IA, Couraud PO. The hCMEC/D3 cell line as a model of the human blood brain barrier. Fluids Barriers CNS. 2013;10(1):16.

21. Mizee MR et al. Astrocyte-derived retinoic acid: a novel regulator of blood-brain barrier function in multiple sclerosis. Acta Neuropathol. 2014;128(5):691-703.

22. Lopez-Ramirez MA, Reijerkerk A, de Vries HE, Romero IA. Regulation of brain endothelial barrier function by microRNAs in health and neuroinflammation. FASEB J. 2016;30(8):2662-2672.

23. Vogel DY et al. GM-CSF promotes migration of human monocytes across the blood brain barrier. Eur J Immunol. 2015; 45(6):1808-1819.

24. Kooij G, Mizee MR, van Horssen J et al Adenosine triphosphate-binding cassette transporters mediate chemokine (C-C motif) ligand 2 secretion from reactive astrocytes: relevance to multiple sclerosis pathogenesis. Brain. 2011;134(Pt 2):555570 .

25. Lublin FD, Reingold FC, Cohen JA et al. Defining the clinical course of multiple scle- 
rosis: the 2013 revisions. Neurology. 2014;83(3):278-286.

26. Reich DS, Lucchinetti CF, Calabresi PA. Multiple Sclerosis. N Engl J Med. 2018; 378(2):169-180.

27. Chiurchiù V. Novel targets in multiple sclerosis: to oxidative stress and beyond. Curr Top Med Chem. 2014;14(22):2590-2599.

28. Prüss H, Rosche $B$, Sullivan $A B$ et al. Proresolution lipid mediators in multiple sclerosis - differential, disease severity dependent synthesis - a clinical pilot trial. PLoS One. 2013;8(2):e55859.

29. Hsiao H-M, Thatcher TH, Colas RA, Serhan CN, Phipps RP, Sime PJ. Resolvin D1 reduces emphysema and chronic inflammation. Am J Pathol. 2015;185:318931201.

30. Hellmann J, Tang Y, Kosuri M, Bhatnagar A, Spite M. Resolvin D1 decreases adipose tissue macrophage accumulation and improves insulin sensitivity in obese-diabetic mice. FASEB J 2011;25:2399-23407.

31. Clària J, Dalli J, Yacoubian S, Gao F, Serhan $\mathrm{CN}$. Resolvin D1 and resolvin D2 govern local inflammatory tone in obese fat. J Immunol. 2012;189:2597-25605

32. Bento AF, Claudino RF, Dutra RC, Marcon $\mathrm{R}$, Calixto JB. Omega-3 fatty acid-derived mediators 17(R)-hydroxy docosahexaenoic acid, aspirintriggered resolvin D1 and resolvin D2 prevent experimental colitis in mice. J Immunol. 2011;187:1957-1969.

33. Schwanke RC, Marcon $R$, Bento AF Calixto JB. EPA- and DHA-derived resolvins' actions in inflammatory bowel disease. Eur J Pharmacol. 2016;785:156164.

34. Perretti M, Norling LV. Actions of SPM in regulating host responses in arthritis. Mol Aspects Med. 2017;58:57-64.

35. Fredman G, Hellmann J, Proto JD, Kuriakose G, Colas RA, Dorweiler B, et al. An imbalance between specialized proresolving lipid mediators and pro-inflammatory leukotrienes promotes instability of atherosclerotic plaques. Nat Commun. 2016;7:12859.

36. Wang X, Zhu M, Hjorth E, Cortés-Toro V, Eyjolfsdottir H, Graff C, et al. Resolution of inflammation is altered in Alzheimer's disease. Alzheimers Dement. 2015;11:40.e50.e.

37. Zhu M, Wang X, Hjorth E, Colas RA, Schroeder L, Granholm A-C, et al. Pro- resolving lipid mediators improve neuronal survival and increase A 42 phagocytosis. Mol Neurobiol. 2016;53:2733-2749.

38. Fiala M, Halder RC, Sagong B, Ross O, Sayre J, Porter V, et al. -3 supplementation increases amyloid- phagocytosis and resolvin D1 in patients with minor cognitive impairment. FASEB J. 2015;29:26812689.

39. Holman RT, Johnson SB, Kokmen E. Deficiencies of polyunsaturated fatty acids and replacement by nonessential fatty acids in plasma lipids in multiple sclerosis. Proc Natl Acad Sci U S A. 1989;86(12):47204724.

40. Poisson LM, Suhail H, Singh J et al. Untargeted plasma metabolomics identifies endogenous Metabolite with Drug-like properties in chronic animal model of multiple sclerosis. J Biol Chem. 2015; 290(52):30697-30712.

41. Jelinek GA Hadgkiss EJ, Weiland TI Pereira NG, Marck CH, van der Meer DM Association of fish consumption and $\Omega 3$ supplementation with quality of life, disability and disease activity in an international cohort of people with multiple sclerosis. Int J Neurosci. 2013;123(11):792 800.

42. Weinstock-Guttman B, Baier M, Park Y et al. Low fat dietary intervention with omega-3 fatty acid supplementation in multiple sclerosis patients. Prostaglandins Leukot Essent Fatty Acids. 2005;73(5):397404.

43. Reder AT, Genc K, Byskosh PV, Porrini AM. Monocyte activation in multiple sclerosis. Mult Scler 1998; 4: 162-168.

44. Chuluundorj D, Harding SA, Abernethy D, La Flamme AC. Expansion and preferentia activation of the CD14(+)CD16(+) monocyte subset during multiple sclerosis. Immunol Cell Biol. 2014;92(6):509-517.

45. Singh A et al. Lipoxin A4, a 5-lipoxygenase pathway metabolite, modulates immune response during acute respiratory tularemia. J Leukoc Biol. 2017;101(2):531542.

46. Gu Z, Lamont GJ, Lamont RJ, Uriarte SM, Wang H, Scott DA. Resolvin D1, resolvin D2 and maresin 1 activate the GSK3 antiinflammatory axis in TLR4-engaged human monocytes. Innate Immun. 2016;22(3):186195.

47. Koltsida O, Karamnov S, Pyrillou K et al.
Toll-like receptor 7 stimulates production of specialized pro-resolving lipid mediators and promotes resolution of airway inflammation. EMBO Mol Med. 2013;5(5):762 775

48. Ortiz GG, Pacheco-Moisés FP, Macías-Islas MA et al. Role of the blood-brain barrier in multiple sclerosis. Arch Med Res. 2014; 45(8):687-697.

49. Kadl A, Leitinger N. The role of endothelial cells in the resolution of acute inflammation. Antioxid Redox Signal. 2005;7(11 12):1744-1754.

50. Chattopadhyay R, Raghavan S, Rao GN Resolvin D1 via prevention of ROS-mediated SHP2 inactivation protects endothelial adherens junction integrity and barrier function. Redox Biol. 2017;12:438-455.

51. Zhang X, Wang Y, Gui P, et al. Resolvin D1 reverts lipopolysaccharide-induced TJ proteins disruption and the increase of cellular permeability by regulating I B signaling in human vascular endothelial cells. Oxid Med Cell Longev. 2013;2013:185715.

52. Pang $\mathrm{H}$, Huang YP, Liu ZJ, et al. Effect of lipoxin A4 on lipopolysaccharide-induced endothelial hyperpermeability. Scientific World Journal. 2011:11:1056-1067.

53. Jones CN, Dalli J, Dimisko L, Wong E, Serhan CN, Irimia D. Microfluidic chambers for monitoring leukocyte trafficking and humanized nano-proresolving medicines interactions. Proc Natl Acad Sci U S A 2012;109(50):20560-20565.

54. Akagi D, Chen M, Toy R, Chatterjee A Conte MS. Systemic delivery of proresolving lipid mediators resolvin D2 and maresin 1 attenuates intimal hyperplasia in mice. FASEB J. 2015;29(6):2504-2513.

55. Baker N, O'Meara SI, Scannell M, Maderna P, Godson C. Lipoxin A4: anti-inflammatory and anti-angiogenic impact on endothelial cells. J Immunol. 2009:182(6):3819-26.

56. Chinthamani S, Odusanwo O, Mondal N, Nelson J, Neelamegham S, Baker OJ Lipoxin A4 inhibits immune cell binding to salivary epithelium and vascular endothelium. Am J Physiol Cell Physiol. 2012 302(7):C968-C978.

57. Merched AJ, Ko K, Gotlinger KH, Serhan $\mathrm{CN}$, Chan L. Atherosclerosis: evidence for impairment of resolution of vascular inflammation governed by specific lipid mediators. FASEB J. 2008;22(10):3595606. 